\title{
CARACTERÍSTICAS ESPECTRO-TEMPORAIS DE CORRENTES TIPO I
}

\section{SPECTRO-TEMPORAL CHARACTERISTICS FROM CHAINS OF TYPE I}

\author{
Zuleika Auxiliadora da Luz Sodré ${ }^{1}$ \\ Francisco Carlos Rocha Fernandes ${ }^{2}$
}

RESUMO: Correntes tipo I são rádio emissões solares que apresentam aparência complexa e sequências com pelo menos 4 emissões tipo I individuais (banda estreita e curta duração), com separação inferior a 1 segundo e, aproximadamente ( $\pm 5 \%)$, a mesma frequência central. Com base nas características morfológicas identificadas nos espectros dinâmicos registrados pelo CALLISTO-BLEN, na faixa de frequências (170-870 MHz), no período de 30 de julho a 09 de agosto de 2011, foram selecionadas 225 correntes tipo I métricas. A análise das características espectro-temporais dessas correntes aponta: frequência central na faixa de 200 a 400 MHz; largura de banda de 1 a $150 \mathrm{MHz}$; duração total de 20 a 600 segundos e deriva em frequência na faixa de -3 a +3 MHz/s.

Palavras-chave: emissões solares; ondas métricas; correntes tipo I; CALLISTO-BLEN.

ABSTRACT: Chains of type I are solar radio emissions that present complex appearance and sequences with at least 4 individual type I emissions (narrow band and short duration) with separation of less than 1 second and approximately $( \pm 5 \%)$ the same central frequency. Based on morphological characteristics identified in dynamic spectra recorded by CALLISTO-BLEN, in the frequency band (170-870 MHz) in the period from July 30 to August 9, 2011, 225 chains of type I metrics were selected. The analysis of the spectro-temporal characteristics of the chains indicates: the central frequency in the range of 200 to $400 \mathrm{MHz}$; the frequency band of 1 to $150 \mathrm{MHz}$; the total duration of 20 to 600 seconds; and the frequency drift rate in the range of -3 to $+3 \mathrm{MHz} / \mathrm{s}$.

Keywords: solar emissions; metric wavelengths; chains of type I; CALLISTO-BLEN.

\footnotetext{
1 Doutoranda em Física e Astronomia - Universidade do Vale do Paraíba - Univap / Instituto de Pesquisa e Desenvolvimento IP\&D. E-mail: zuleika.sjc@bol.com.br.

${ }^{2}$ Doutor em Astrofísica - INPE e Docente da Univap / IP\&D - Laboratório de Física e Astronomia. E-mail: guga@univap.br.

Revista Univap - Edição Especial - revista.univap.br

São José dos Campos-SP-Brasil, v. 19, n. 34, nov.2013. ISSN 2237-1753
} 


\section{INTRODUÇÃO}

Pela definição de Kai, Melrose e Suzuki (1985), uma tempestade é uma rádio emissão solar de longa duração em comprimento de onda métrico ou decamétrico, composta de milhares de bursts de curta duração, sobrepostos a um contínuo de variação lenta, sendo que a longa duração é uma das características que distingue tempestades de outros tipos de emissão solar em rádio, ou seja, têm duração de poucas horas a vários dias.

Um burst é uma emissão isolada, o fenômeno de menor duração observado em uma emissão solar em ondas de rádio; fenômeno básico de uma tempestade de ruído (ELGAROY, 1961).
Durante uma tempestade de ruído, conhecida também como tempestade tipo I, bursts de tipo são emitidos em grande número e quando ocorrem sucessivamente são denominados correntes. De acordo com Elgaroy e Ugland (1970), por definição uma corrente deve apresentar as seguintes características: conter pelo menos 4 bursts, separação inferior a 1 segundo entre bursts individuais e, aproximadamente, a mesma frequência central $( \pm 5 \%)$ para todos os bursts da corrente. Para ilustrar essas definições, na Figura 1 é apresentado um espectro dinâmico (frequência $X$ tempo $X$ intensidade) de emissões caracterizadas como burst, corrente e tempestade tipo I.

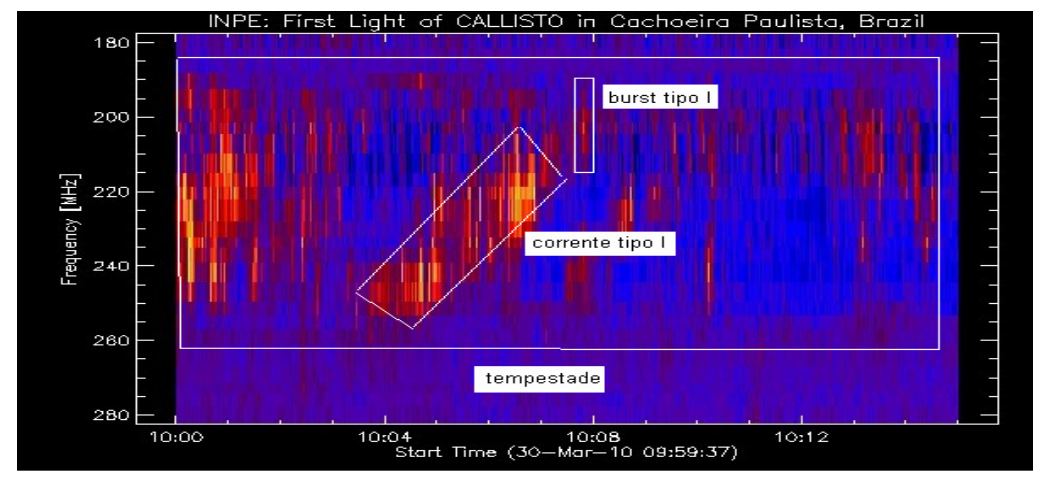

Figura 1 - Exemplo de um espectro dinâmico ilustrando um burst tipo I, uma corrente tipo I e uma tempestade.

Fonte: Adaptado de http://e-callisto.blogspot.com.br/2010_04_01 archive.html.

A primeira investigação mais detalhada sobre correntes foi publicada em 1965, por Wild e Tlamicha, seguidos por outros autores, entre eles, Elgaroy e Ugland (1970), Markeev e Chernov (1971), entre outros (DE GROOT; LOONEN; STOTTJE, 1976).
Hanasz (1966), analisando características de correntes, não encontrou distinção entre as propriedades físicas das mesmas e de bursts individuais, assim, neste trabalho, as mesmas definições e propriedades dos bursts individuais são utilizadas na análise das correntes. 


\section{SELEÇÃO DOS DADOS}

Com o intuito de investigar suas características espectros-temporais, foram identificadas e analisadas 225 correntes tipo I, presentes nos dados em ondas métricas de observações solares realizadas pelo CALLISTO-BLEN, às 04:30 UT às 18:30 UT, entre os dias 30 de julho e 09 de agosto de
2011. Os critérios de seleção das correntes tipo I são descritos por Sodré (2013).

As características determinadas foram: largura de banda $(\Delta \mathrm{B})$, tempo de vida (duração) $(\Delta \mathrm{t})$ e deriva em frequência $(\Delta \mathrm{f} / \Delta \mathrm{t})$, que podem ser vistas na Figura $2 \mathrm{e}$ são descritas, nas seções, a seguir.

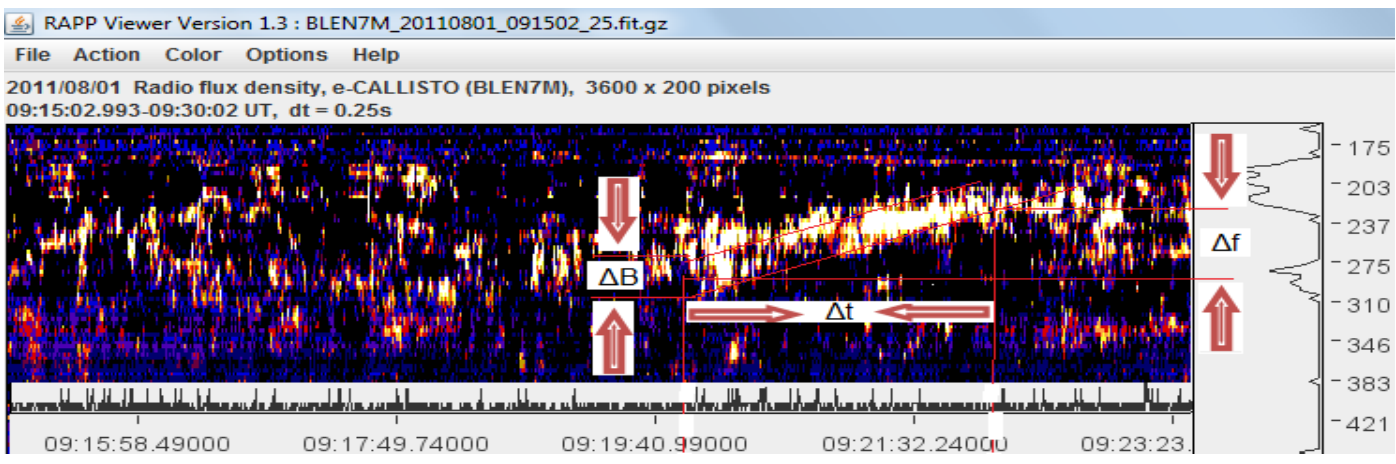

Figura 2 - Características determinadas das correntes tipo I, largura de banda $(\Delta B)$, tempo de vida $(\Delta t)$ e frequência $(\Delta f)$, mostradas no espectro dinâmico do espectrógrafo BLEN7M, no dia 01 de agosto de 2011, entre 09:15 UT e 09:23 UT.

Fonte: Adaptado de http://soleil.i4ds.ch/solarradio/data/2002-20yyCallisto/2011.

\section{RESULTADOS E DISCUSSÃO}

\subsection{Largura de Banda}

A determinação da largura de banda de cada corrente tipo I foi realizada seguindo o critério sugerido por De Groot, Loonen e Slottje (1976), ou seja, ajustando uma reta na parte inferior e outra na superior de cada corrente, abrangendo, pelo menos, $90 \%$ do evento.

A análise aponta que $60 \%$ das correntes apresentam largura de banda entre 15 e $35 \mathrm{MHz}$.

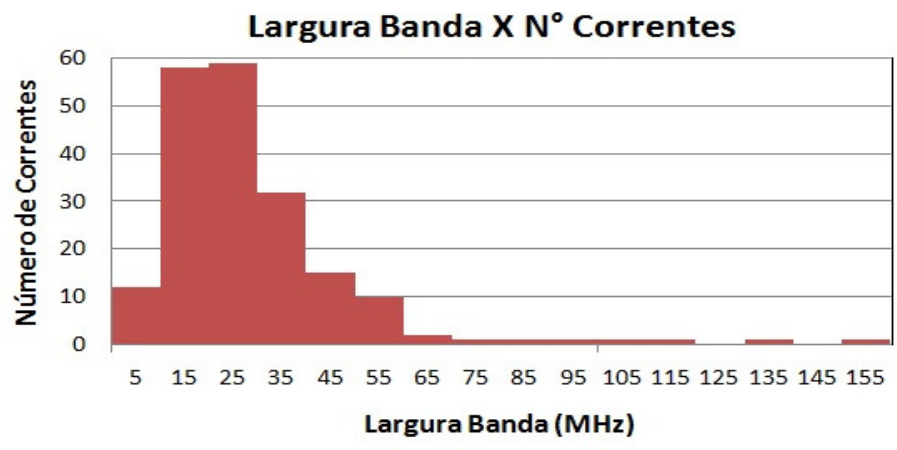

Figura 3 - Distribuição das correntes tipo I em função da largura de banda.

Revista Univap - Edição Especial - revista.univap.br

São José dos Campos-SP-Brasil, v. 19, n. 34, nov.2013. ISSN 2237-1753 
Segundo Kai, Melrose e Suzuki (1985), a largura de banda de tempestades tipo I encontra-se desde algumas dezenas de $\mathrm{MHz}$ até, aproximadamente, $100 \mathrm{MHz}$, sugerindo, assim, que a fonte da tempestade encontra-se confinada em uma faixa de altura relativamente pequena na coroa, e que a largura extremamente estreita dos bursts individuais, em uma tempestade, pressupõe a ocorrência da aceleração dos elétrons em uma pequena distância na região de origem desse fenômeno solar.

Segundo Elgaroy e Eckhoff (1966), a largura de banda de bursts pode ser devido à variação da densidade eletrônica através da fonte e a dispersão na velocidade do feixe.

\subsection{Duração}

A duração total para cada corrente tipo I, referida por diversos autores, entre eles Hanasz (1966) e Elgaroy e Ugland (1970), como o tempo de vida da corrente, foi medido neste trabalho como sendo a diferença entre os instantes de início e fim da emissão, passíveis de identificação com relação ao sinal de fundo (background).

As correntes analisadas apresentaram duração total entre aproximadamente $20 \mathrm{e}$ 600 segundos, sendo que $63 \%$ delas se apresentam entre 50 e 200 segundos, o que concorda com a definição de Elgaroy e Ugland (1970) de que as correntes de longa duração são menos frequentes do que as de curta duração.

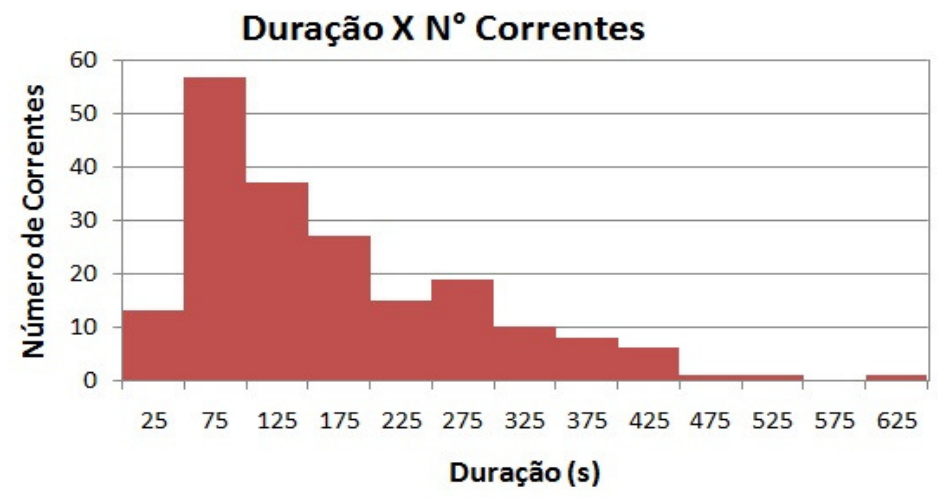

Figura 4 - Distribuição das correntes tipo I em função da duração total.

Para Takakura (1963), a duração do burst é determinada pelo tempo que um feixe de elétrons excita as ondas de plasma. O tempo de vida médio de bursts individuais aumenta com a diminuição da frequência e isso parece implicar no fato de que elétrons acelerados perdem sua energia numa curta distância (KAI; MELROSE; SUZUKI, 1985).

\subsection{Deriva em Frequência}

Uma das principais características espectros-temporais apresentada pela maioria das correntes tipo I é uma sistemática taxa de deriva em frequência.

Das 225 correntes, aqui analisadas, 133 (59,11\%) aparecem com deriva em frequência entre $-0,5$ e $0 \mathrm{MHz} / \mathrm{s}$. 


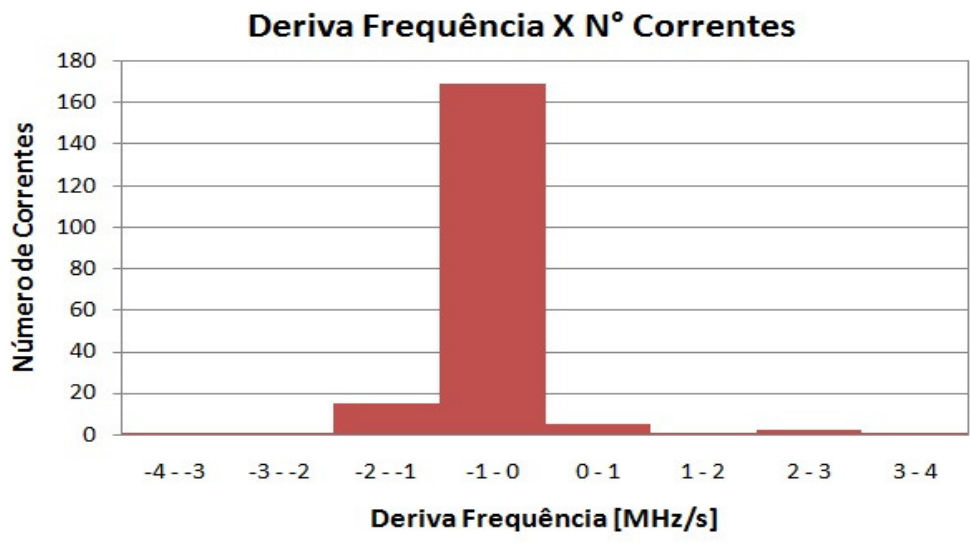

Figura 5 - Distribuição das correntes tipo I em função da taxa de deriva em frequência.

Segundo Wild e Tlamicha (1964), a deriva de frequência observada em correntes tipo I corresponde ao movimento de distúrbios na coroa.

Elgaroy (1961) encontrou que bursts tipo I podem apresentar deriva de frequência positiva ou negativa, e uma única tempestade pode conter uma mistura de bursts com deriva positiva e negativa. Esses resultados sugerem que os elétrons acelerados responsáveis pelos bursts podem fluir para cima ou para baixo na região de origem, ou podem ser aprisionados num nível de frequência de plasma constante.

\section{CONCLUSÃO}

Neste trabalho, foram analisados dados de 225 correntes tipo I, registradas pelo CALLISTO-BLEN, no período de 30 de julho a 09 de agosto de 2011, seguindo uma metodologia adaptada de estudos prévios descritos na literatura, como os de Elgaroy e Ugland (1970) e De Groot, Loonen e Slottje (1976), tendo, como base, a determinação das características espectro-temporais registradas na forma de espectros dinâmicos para as correntes tipo I. A análise estatística dos resultados mostrou que os valores determinados concordam, em grande parte, com os reportados anteriormente na literatura, como os de Wild e Tlamicha (1964) e Elgaroy e Ugland (1970), entre outros. Devido às diferenças instrumentais e metodológicas deste trabalho, em relação aos citados anteriormente, os resultados obtidos, apesar de representar uma amostra menor, mostram-se representativos para a faixa de ocorrência das emissões tipo I. Também auxiliam no avanço do entendimento das emissões tipo I, uma vez que estas podem servir de diagnóstico de diversos processos físicos do Sol e são ainda as menos estudadas.

\section{AGRADECIMENTOS}

Z. A. L. Sodré agradece a bolsa de Doutorado concedida pela Capes e à Fundação Valeparaibana de Ensino (FVE), pela bolsa-mensalidade. F. C. R. Fernandes agrade ao CNPq, pela Bolsa de Produtividade em Pesquisa (Proc. $\mathrm{n}^{\circ}$ 308755/2012-0). 


\section{REFERÊNCIAS}

DE GROOT, T.; LOONEN, J.; SLOTTJE, C. Chains of type I stormbursts. Sol. Phys., v. 48, p. 321-337, 1976.

ELGAROY, O. High resolution spectrometry of enhanced solar radio emission. Astrophysica Norvegica, v. 7, p. 123- 261, 1961.

ELGAROY, O.; ECKHOFF, H. Kr. On the active region corona and the interpretation of type I burst. Astrophysica Norvegica, v. 10, p. 127-148, 1966.

ELGAROY, O.; UGLAND, O. Characteristics properties of small chains of type I solar radio bursts. Astron. Astrophys., v. 5, p. 372381, 1970.

HANASZ, J. Chains of type I solar radio burst. Aust. J. Phys., v. 19, p. 635-647, 1966.

KAI, K.; MELROSE, D.; SUZUKI, S. Solar radiophysics-studies of emission from the sun at metre wavelengths. $1^{\text {st }}$ ed., Cambridge: Press Syndicate of the University of Cambridge, 1985.
MARKEEV, A. K.; CHERNOV, G. P. Observations of Solar Radio Bursts with High Spectral Resolution. Soviet Astronomy, v. 14, p. $835,1971$.

SODRÉ, Z. A. L. Identificação e Análise de Correntes Tipo I Métricas Registradas pelo CALLISTO-BLEN no Período de 30 de julho a 09 de agosto de 2011, 2013. Dissertação (Mestrado em Física e Astronomia) - Instituto de Pesquisa e Desenvolvimento. Universidade do Vale do Paraíba, São José dos Campos, 2013.

TAKAKURA, G. Origin of Solar Radio Type I Bursts. Astronomical Society of Japan, v. 15, p. 462-467, 1963.

WILD, J. P.; TLAMICHA, A. Spectral evidence on the origin of type I solar radio storms and the coronal magnetic field. Nature, v. 203, p. 1128, 1964.

Spectral evidence on the origin of type I solar radio storms and the coronal magnetic field. Bulletin of the Astronomical Institute of Czechoslovakia, v. 16, n. 2, p. 7376, 1965. 